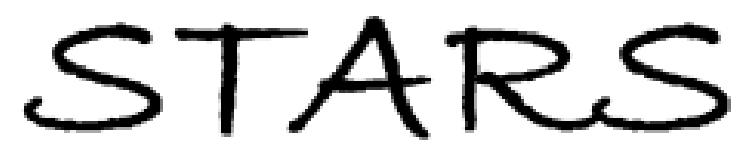

University of Central Florida

STARS

Faculty Bibliography 2010s

Faculty Bibliography

$1-1-2010$

\title{
Silicon-photonics-based wideband radar beamforming: basic design
}

Sasan Fathpour

University of Central Florida

Nabeel A. Riza

University of Central Florida

Find similar works at: https://stars.library.ucf.edu/facultybib2010

University of Central Florida Libraries http://library.ucf.edu

This Article is brought to you for free and open access by the Faculty Bibliography at STARS. It has been accepted for inclusion in Faculty Bibliography 2010 s by an authorized administrator of STARS. For more information, please contactSTARS@ucf.edu.

\section{Recommended Citation}

Fathpour, Sasan and Riza, Nabeel A., "Silicon-photonics-based wideband radar beamforming: basic design" (2010). Faculty Bibliography 2010s. 147.

https://stars.library.ucf.edu/facultybib2010/147

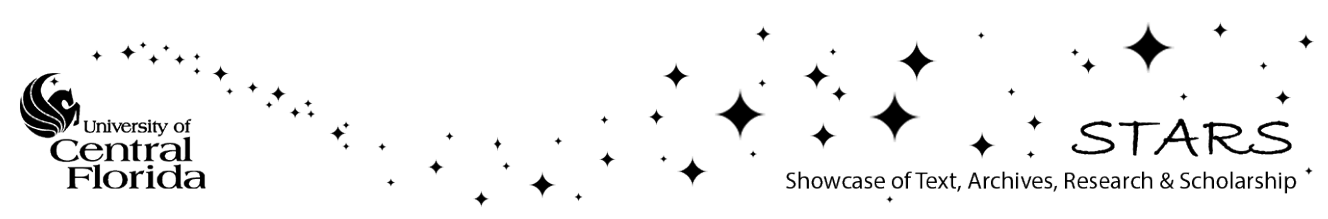




\section{Silicon-photonics-based wideband radar beamforming: basic design}

\author{
Sasan Fathpour, MEMBER SPIE \\ Nabeel A. Riza, FELLOW SPIE \\ University of Central Florida \\ CREOL, The College of Optics and Photonics \\ 4000 Central Florida Boulevard \\ Orlando, Florida 32816-2700 \\ E-mail: fathpour@creol.ucf.edu
}

\begin{abstract}
Proposed is silicon-photonics-based phased array antenna beamforming for high-resolution long-range radars with wide instantaneous radio frequency (rf) bandwidth. Specifically, the proposed siliconphotonics beamformer platform offers the potential for cost-effective monolithic chip-scale integration of photonic delay lines, $2 \times 2$ optical switches, variable optical attenuators, and optical amplifiers that form the base unit of a rf transmit/receive array signal processor. In effect, the proposed silicon-photonics devices empower the design of a powerful proposed photonic beamformer with one time-delay unit per antenna element. Device-level designs studies are shown that promise meeting the high-resolution radar mission-critical requirements via time delays of up to $2.5 \mathrm{~ns}$, switching times of less than $100 \mathrm{~ns}$, optical isolations as good as $50 \mathrm{~dB}$, and optical gains of up to $6 \mathrm{~dB}$. Longer delays are achieved off chip using optical fibers. @ 2010 Society of Photo-Optical Instrumentation Engineers. [DOI: 10.1117/1.3280286]
\end{abstract}

Subject terms: Fourier optics and signal processing; analog optical signal processing; continuous optical signal processing.

Paper 090573R received Jul. 28, 2009; revised manuscript received Nov. 5 , 2009; accepted for publication Nov. 11, 2009; published online Jan. 7, 2010.

\section{Introduction}

Photonics has long been recognized as a viable technology for distribution and processing of millimeter and microwave signals in modern phased-array antennas (PAAs)! 1 Advantages of photonics over electronics for these beamforming control systems include reduced weight and size, immunity to electromagnetic interference (EMI) and electromagnetic pulses (EMP), and low rf transmission loss. In particular, photonic technologies have been successfully employed to demonstrate true time-delay beam steering, which is essential for squint-free wide-instantaneousbandwidth operation of high-performance radar systems.

A typical advanced wideband radar beamforming system design is shown in Fig. 11 where each antenna element uses an independent rf signal-conditioning unit. Note that a beamformer is a complex interconnection of $\mathrm{rf}$ waveguides (splitters and combiners) that can produce different timedelay errors per antenna element depending on system conditions such as the beamformer temperature and stresses and strains in different parts of the waveguides. Therefore, any robust beamformer architecture such as the Fig. 11 design involves an independently controllable time-delay unit (TDU) per antenna element. Electronically, a beamformer system includes high-power amplifiers (HPAs), low-noise amplifiers (LNAs), rf attenuators, and transmit/receive $(\mathrm{T} / \mathrm{R}) \mathrm{rf}$ switches. In addition, if optical fibers are deployed in the rf signaling control system, such as for distribution and rf-optical-rf links, then again temperature changes and stresses can put strains in the fibers that can independently affect the different rf time-delay paths in the beamforming network. Thus, any practical deployed wide-instantaneousbandwidth radar beamformer for demanding outdoor environments must use an independently controllable TDU per antenna element, and this TDU should produce both long delays (e.g., $10 \mathrm{~ns}$ ) and high-resolution delays (e.g., 10 ps), equivalent to 1000 settings, or 10 bits. In addition, the TDU should have other general features such as wideband rf operation [e.g., S/C band 2 to $8 \mathrm{GHz}$ or $\mathrm{X}$ band (8 to $12 \mathrm{GHz}$ )], low loss (e.g., $<5 \mathrm{~dB}$ optical), low crosstalk (e.g., $<5$ ) dB optical), and fast (submicrosecond time delay) reset? To date, such a desired TDU is altogether lacking in the all-electronic domain.

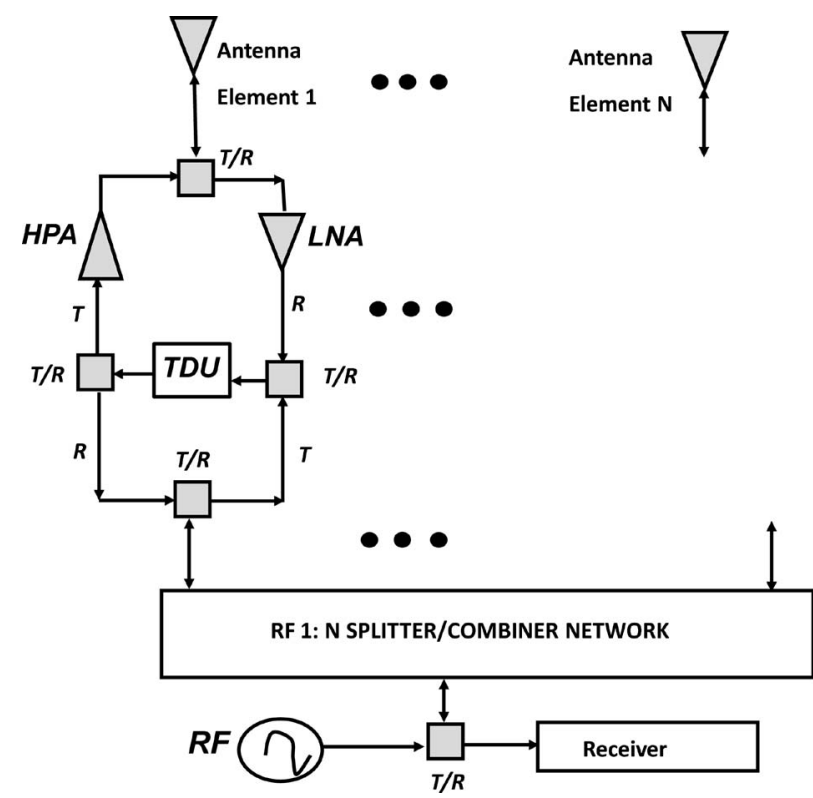

Fig. 1 An advanced if PAA beamformer design using one variable TDU per antenna element. HPA: high-power amplifier; LNA: lownoise amplifier; T/R: rf transmit/receive switch; TDU: time-delay unit with if in and if out. 
A wide variety of schemes and device technologies have been proposed and used for realizing optical beamformers,,$[25$ including silica glass, III-V semiconductors, polymers, electromechanical and microelectromechanical systems (MEMS), lithium niobate, and nematic and ferroelectric liquid crystals. However, these proposed and implemented systems do not meet the requirements mentioned for advanced TDUs. Recently, it was shown that acoustooptic switching 26 can indeed meet the desired TDU requirements, but this device technology, like many others before it, is presently limited in capability of cost-effective scaling to practical PAA TDU counts (e.g., >500) required in large PAAs. Given this motivation, this paper uses silicon photonics as the base platform to propose the desired design of a superperformance (e.g., 10 bits), superfast (e.g., $<1 \mu \mathrm{s}$ ) optical TDU design that can realize the wideband PAA radar control mission. Shown are basic device design and simulation studies indicating the performance expected from the proposed silicon photonic TDUs.

Many years ago, silicon photonics was proposed for optical switching at $1300 \mathrm{~nm} .27$ Meanwhile, silicon photonics has matured in the last few years as a viable technology for a host of applications, particularly for passive and active integrated optics 28 Silicon-based delay lines with subpicosecond time-delay accuracy have been demonstrated, although with very short time delays of the order of tens of picoseconds 29 A new CMOS-compatible material named Hydex ${ }^{\circledR}$, allowing delays as high as $2 \mathrm{~ns}$, has also been reported 30 Compared to competing technologies based on nonlinearity in fibers and the electro-optic (EO) effect in lithium niobate and III-V semiconductors, silicon photonics has several key advantages, which include:

(a) Cost-effectiveness: Lots (e.g., thousands) of TDUs per large-aperture advanced phased-array radar (e.g., Aegis class) are required, particularly for a fully agile three-dimensional (3-D) electronic or e-scan system with complete beam power. A monolithic technique capable of processing all these TDUs simultaneously would substantially reduce manufacturing costs.

(b) CMOS compatibility: Unlike competing materials, silicon has the great advantage of compatibility with silicon integrated circuit (IC) technology required to electrically drive the devices. In addition, compatibility of silicon photonics with CMOS processing and integration with variable optical attenuators (VOAs) and rf-to-optical modulators also implies further cost reduction and high-yield manufacturing.

(c) Compactness: The high refractive index contrast between silicon and $\mathrm{SiO}_{2}$ provides a platform for submicron waveguide dimensions in silicon-oninsulator (SOI) waveguides, leading to a smaller footprint and reduced weight for a silicon photonic beamformer.

(d) Integration: Compact $(\sim 1 \mathrm{~cm})$, fast $(<1 \mu \mathrm{s})$, high-attenuation $(>40 \mathrm{~dB})$ VOAs have been recently demonstrated 31 and can be monolithically integrated with the optical switches, photonic delay lines, and optical amplifiers of the proposed TDU. In addition, the rf-to-optical modulator that provides the PAA's rf signal can also be integrated on the same silicon chip, a feature presently hard to achieve in the competing material technologies.

Another vital component of an optical TDU is a highspeed rf-band photodetector. Because of its optical transparency in the 1300 and 1550-nm wavelength bands typically used for rf EO modulation, silicon is not a suitable material for rf demodulation via photodetection. With a smaller bandgap, germanium $(\mathrm{Ge})$ has strong absorption at these wavelengths, and Ge photodetectors can be integrated on silicon chips. High-performance hybrid $\mathrm{Ge}(\mathrm{Si})$-on-Si photoreceivers have been demonstrated ${ }^{28}$ for example, a Ge-on-SOI photodetector with a $6.6-\mathrm{GHz}$ bandwidth

\section{Proposed Beamsteering System}

Figure 2 shows the proposed basic silicon-based TDU for implementing PAA beamsteering. The unit employs a classic serial digitally switched $N$-bit TDU scheme using the proposed silicon $1 \times 2$ and $2 \times 2$ routing switches. Using an $N$-bit delay line, one can produce $2^{N}-1$ independent relative time delays. The delay lines can be on chip, using long integrated waveguides for shorter delays $(<2.5 \mathrm{~ns})$, or can be external optical fibers (for 2.5- to 20-ns delays). One advantage of silicon photonics is that the losses caused per TDU bit stage can be partly compensated by Raman optical amplification. In addition, a VOA can be monolithically integrated on the chip per TDU for signal conditioning required for antenna pattern control as well as rf loss calibration within the beamforming network.

Critical to the design of any TDU is the realization of low-loss, high-speed, low-crosstalk $1 \times 2$ and $2 \times 2$ optical switches to meet the mentioned TDU requirements. Due to the lack of a linear EO effect in silicon, the free-carrier plasma effect has been the most popular mechanism for achieving EO modulation. Specifically, the principle of EO operation involves the modulation of the free-carrier density in which the optical loss and/or refractive index is $\mathrm{rf}$ modulated by injectign, depletion, accumulation, or inversion of free carriers 28 The very first silicon photonic device was a $2 \times 2$ switch based on $n$-type waveguides fabricated on $n^{+}$substrate 27 More recently, EO switches based on SOI substrates have been demonstrated 33 that exhibit high $5-\mathrm{MHz}$ switching speed but lack the TDU's required low (e.g., $>40 \mathrm{~dB}$ ) crosstalk.

To realize the TDU switching goal, Fig. 3(a) shows the proposed novel $1 \times 2$ fast $(<1 \mu \mathrm{s})$ switch based on the free-carrier plasma effect using carrier injection via lateral $p n$ junction diodes that straddle SOI waveguides. The design of these devices is optimized for the targeted beamforming control application, i.e., fast switching time ( $\sim 0.1$ to $1 \mathrm{~ns}$ ) and high on/off extinction ratio, leading to 50 - dB interchannel optical isolations. The proposed $1 \times 2$ switch design uses a silicon Mach-Zehnder interferometer (MZI). At the output of the MZI, the phase-shifted signals are directed into a multimode interference (MMI) coupler that selects the desired output channel. To control the phase-shifting process, refractive index variation is induced by carrier injection (i.e., the plasma effect) via the electrical biases applied to the two pn junction diodes. Shorter de- 


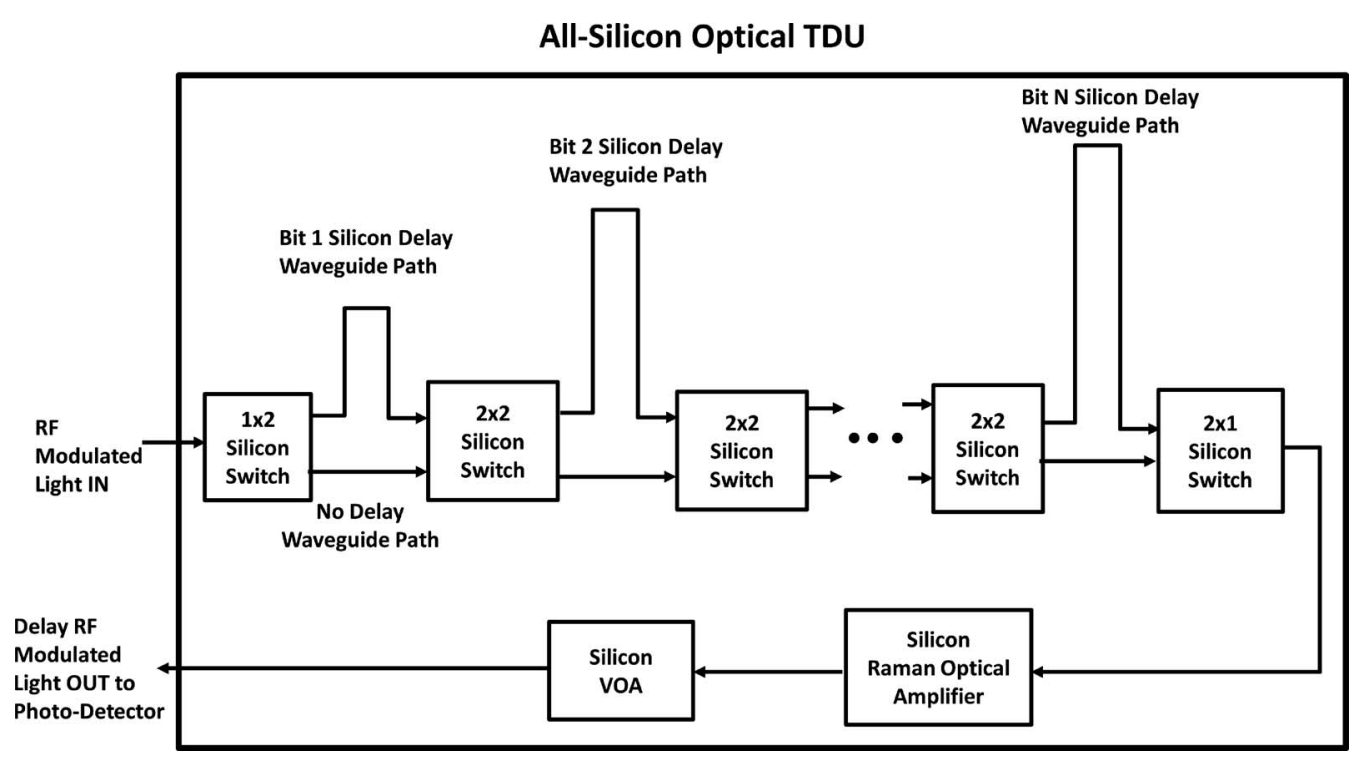

Fig. 2 Proposed $N$-bit programmable silicon photonic TDU module.

vices can be achieved at the expense of higher carrier injection (slower switching operation) via the $p n$ junctions. This trade-off can be optimized according to the specific beamforming system requirements, another useful feature of silicon photonics. Note that low-crosstalk switching cannot be obtained merely based on MMI couplers, and reported optical extinction ratios are on the order of $-22 \mathrm{~dB} 34$ To overcome this shortcoming, the design in Fig.

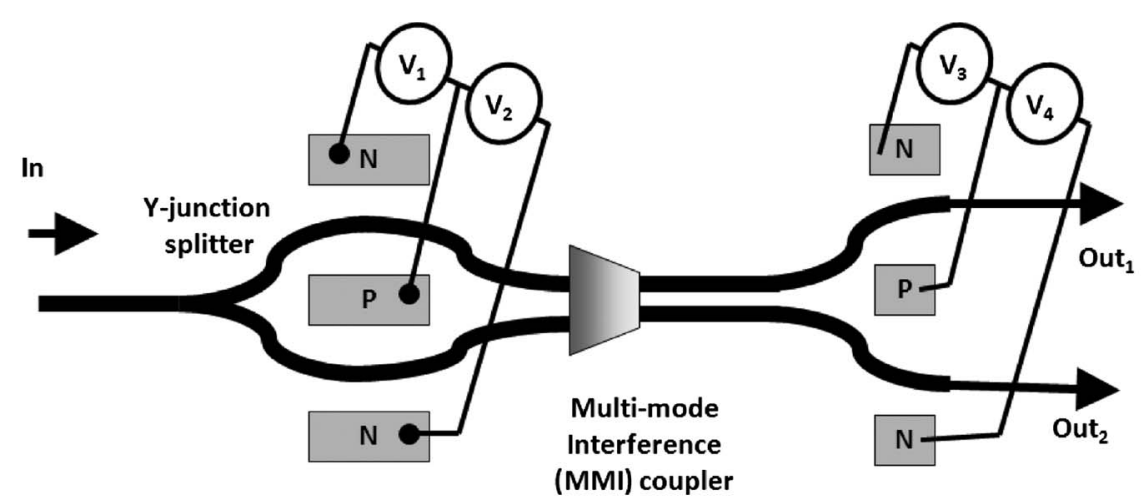

(a)

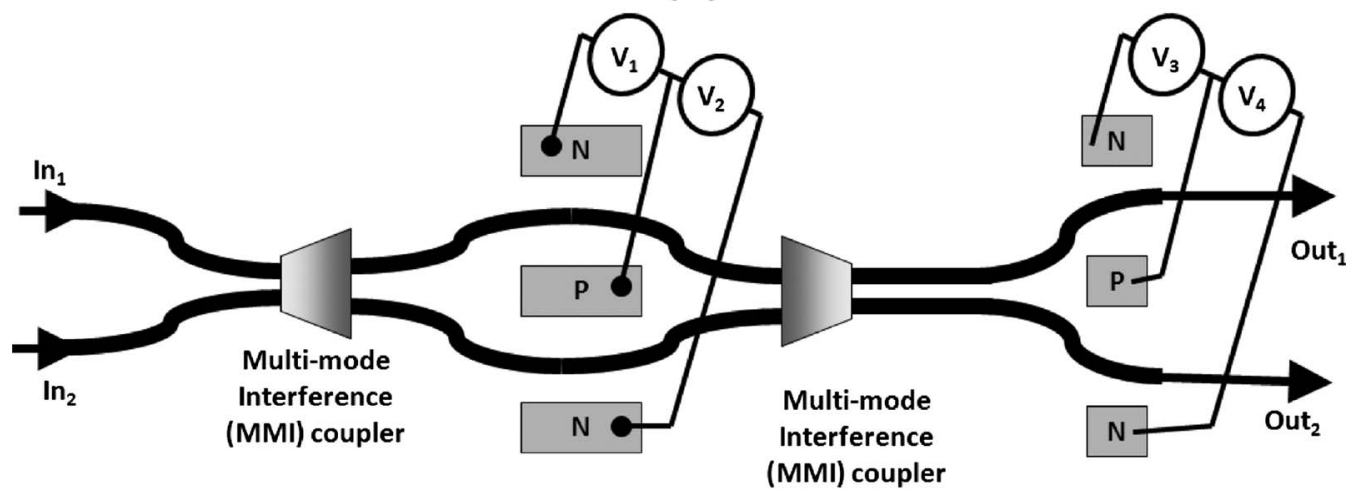

(b)

Fig. 3 Proposed (a) $1 \times 2$ and (b) $2 \times 2$ silicon optical switch designs to be used to realize the TDU design in Fig. 2 


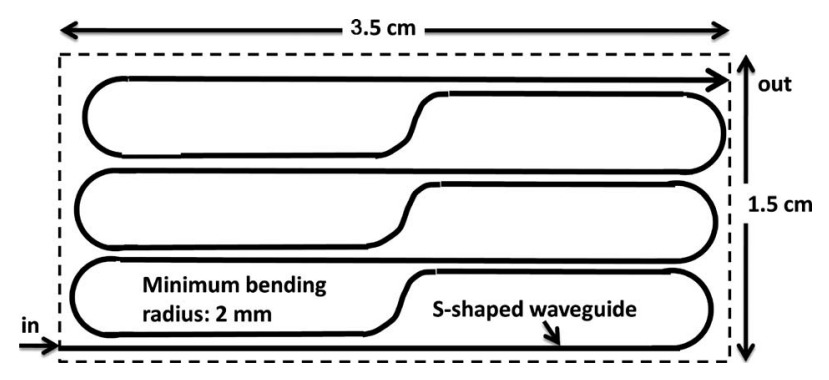

Fig. 4 Proposed S-shaped waveguides for achieving long on-chip photonic delay lines in the silicon TDU of Fig. 2

3(a) proposes inserting two $1 \times 1$ photonic switches at the output arms of the integrated device. Specifically, by applying a forward bias to the off arm, the $1 \times 1$ switch essentially acts like a VOA and can provide an extra $40 \mathrm{~dB}$ or more extinction ratio at high biases, leading to the desired high-isolation photonic switch for routing the no-delay and delay rf signals on the light carrier. It is emphasized that the $p n$ junctions in the MZI arms and those in the output arms serve different purposes. In essence, with the appropriate choice of bias, the same device, consisting of a silicon waveguide with a straddling $p n$ junction, is employed as a phase modulator in the MZI arms, while it is employed as an intensity (loss) modulator in the $1 \times 1$ switches following the MZI.

To realize the desired $2 \times 2$ optical switch required for TDU assembly, a second MMI coupler is inserted at the input of the MZI as depicted in Fig. 3(b) The working principle remains similar to that of the $1 \times 2$ switch. The choice of biases $V_{1}$ and $V_{2}$ allows selecting one of the input ports to be routed to one of the output ports via phase modulation. Similar to the $1 \times 2$ switches, two $1 \times 1$ switches (biases $V_{3}$ and $V_{4}$ ) are inserted at the output arms to provide more than $40-\mathrm{dB}$ extra isolation via intensity (loss) modulation in the off port.

Guided-wave optical delay lines on the TDU silicon chip in Fig. 2 provide high precision for rf beamforming systems, because the waveguides are defined using photolithography, by which waveguide lengths can be controlled with submicron precision with accuracy limits typically set by processing variations or the mask quantization. The higher refractive index of silicon than that of silica $(3.4$ versus 1.45$)$ suggests that a larger time delay per waveguide length $(\approx 12 \mathrm{ps} / \mathrm{mm})$ is possible in silicon. Figure 4 shows how long (2.5-ns) time delays are achievable in a 20-cm-long S-shaped waveguide. The footprint of such a chip is $3.5 \times 1.5 \mathrm{~cm}^{2}$. Note that the proposed silicon photonic delay lines would induce a typical waveguide linear loss of $0.3 \mathrm{~dB} / \mathrm{cm}, 35$ and thus the overall loss can be as high as $6 \mathrm{~dB}$ for a 2.5-ns waveguide delay. Given that beamforming requires cascaded delay lines, a 6-dB loss is considered high. Given that silicon Raman amplifiers have proven successful, 3637 we propose their use for optical loss recovery in the silicon TDU chip. Note that stimulated Raman scattering (SRS) has been long exploited in optical fibers to create amplifiers and lasers. However, several kilometers of fiber are typically required to create a useful device. The gain coefficient for SRS in silicon is approximately $10^{3}$ to $10^{4}$ times higher than that in silica fiber.

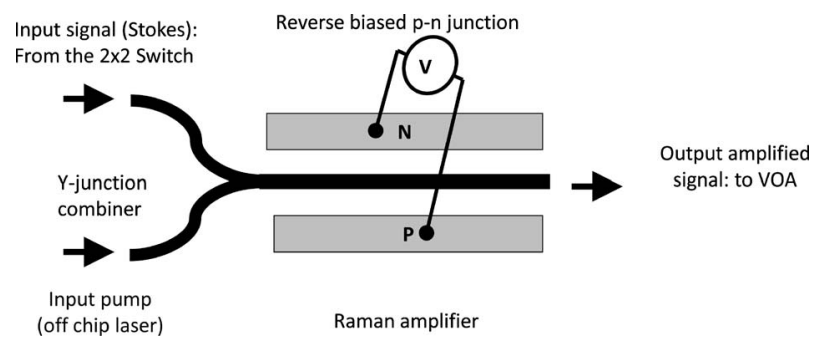

Fig. 5 Proposed method for integrating a photonic delay line and a silicon Raman amplifier needed for the TDU design of Fig. 2

Additionally, silicon waveguides can confine the optical field to an area that is approximately 100 times smaller than the modal area in a standard single-mode optical fiber. Combined, these facts make it possible to observe SRS over the interaction lengths encountered on a chip. To achieve net $\mathrm{cw}$ gain, a $p n$ junction diode can be used to sweep the carriers out of the waveguide core region. Up to $6.5 \mathrm{~dB}$ of optical gain has been demonstrated, 3839 which is sufficient to compensate for the loss of a 2.5-ns silicon waveguide delay. Hence, we propose the integration of such Raman amplifiers in the silicon TDU. For these optically pumped amplifiers, an off-chip laser source is required, whose beam ought to be combined with the rfmodulated signal for the PAA. As shown in Fig. 5 a Y-junction combiner can achieve this goal.

\section{Performance Predictions}

Next, the critical performance parameters of the silicon photonic devices used to realize the proposed TDU are presented. First, the diode waveguides shown in Fig. 3 are analyzed using the lateral geometry shown in Fig. 6. with ridge height $H=2.0 \mu \mathrm{m}$, waveguide width $W=1.5 \mu \mathrm{m}$, slab height $h=1.1 \mu \mathrm{m}$, and ridge-to-contact spacing $d=2 \mu \mathrm{m}$ used in the numerical simulations. The model used in the calculations follows Ref. 40 The dependence of freecarrier absorption (FCA), $\alpha_{\mathrm{FCA}}$, on carrier density is given by

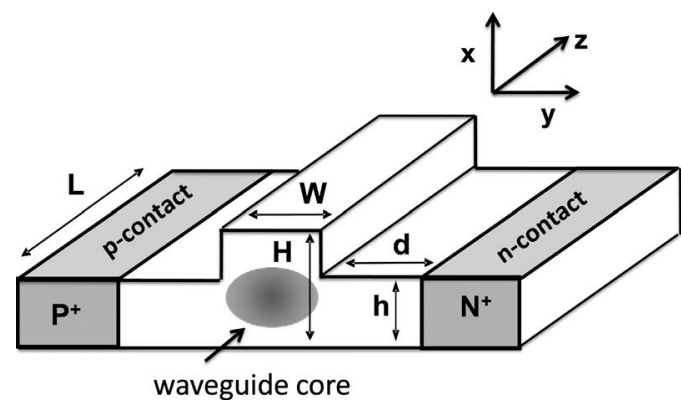

Fig. 6 Schematic of the proposed silicon EO modulator with a straddling pn junction diode. Modulation of carriers (electrons and holes) in the waveguide core region via the $p n$ junction allows phase or intensity modulation of the guided light. Ridge height $H=2.0 \mu \mathrm{m}$, waveguide width $W=1.5 \mu \mathrm{m}$, slab height $h=1.1 \mu \mathrm{m}$, and contact-toridge spacing $d=2 \mu \mathrm{m}$ are used in the numerical simulations. 

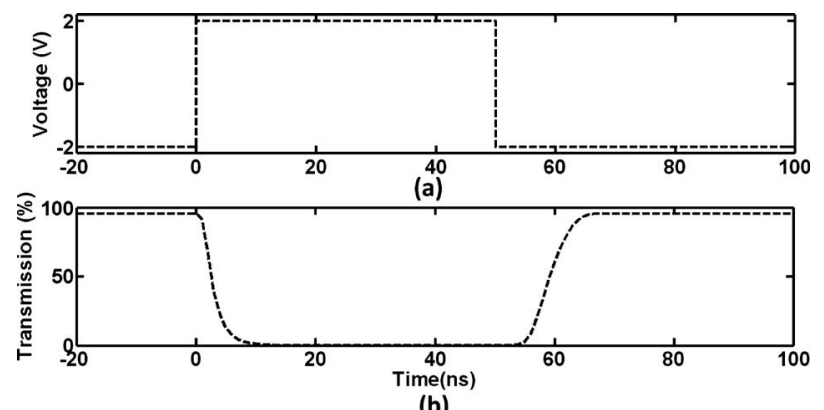

(b)

Fig. 7 Simulated switching results for a silicon intensity (loss) modulator, showing: (a) applied electrical signal; (b) output optical transmission with $\approx 50-\mathrm{dB}$ extinction ratio.

$\alpha_{\mathrm{FCA}}=8.5 \times 10^{-18} \cdot \Delta N+6.0 \times 10^{-18} \cdot \Delta P$,

where $\Delta N$ and $\Delta P$ are the free-electron and hole concentrations, respectively. In the presence of FCA and twophoton absorption (TPA), the propagation of optical intensity $I_{p}(z)$ along the $z$ direction (Fig. 6 is given by the following nonlinear differential equation:

$$
\frac{\mathrm{d} I_{p}(z)}{\mathrm{d} z}=-\left[\alpha+\alpha_{\mathrm{FCA}}(z)\right] I_{p}(z)-\beta I_{p}^{2}(z),
$$

where $\beta=0.7$ centimeter per gigawatt $(\mathrm{cm} / \mathrm{GW})$ is the TPA coefficient, and $\alpha$ is the linear absorption coefficient of the waveguide. Here $\alpha_{\mathrm{FCA}}$ is a function of the optical intensity $I_{p}$ and bias voltage $V$. The device was simulated using a commercial drift-diffusion simulator (ATLAS by Silvaco International). TPA was emulated by specifying a carrier generation rate $G$ at the waveguide core with $G=\mathrm{d} N / \mathrm{d} t=$ $-(1 / 2 E) \mathrm{d} I_{p} / \mathrm{d} z=\beta I_{p}^{2} / 2 E$, where $E=0.8 \mathrm{eV}$ is the photon energy and $N$ is the carrier density. The values of the electron and hole bulk recombination lifetimes were 3 and $10 \mu \mathrm{s}$, respectively. A surface recombination velocity of $200 \mathrm{~cm} / \mathrm{s}$ was used for both types of carriers. The simulator provides the carrier concentration as a function of $V$, from which, by using Eq. (1) the output optical intensity and modulation depth are obtained. The optical transmission and modulation depth can be extracted from $I_{p}(L) / I_{p}(0)$ as a function of $V$. In order to accommodate the 3 -D nature of the problem, a quasi-3D model is developed in which the 2-D results of ATLAS at several optical intensities are interpolated to numerically solve Eq. (2) The simulator also provides the diode current per unit length, $J$ $(\mathrm{A} / \mu \mathrm{m})$.

The switching speed of intensity EO modulators is a key figure of merit for rf photonics. Up to $10-\mathrm{GHz}$ bandwidth has been reported in EO (carrier injection type) silicon optical modulators 41 Figure 7 shows a summary of the conducted switching (intensity modulation) simulation results in a $0.4-\mathrm{cm}-$ long waveguide. A $10-\mathrm{MHz}(100-\mathrm{ns}$ period) square waveform electrical signal with $t_{\text {rise }}=t_{\text {fall }}=200 \mathrm{ps}$ is applied to the diode. An external resistance of $5 \Omega \mathrm{cm}^{2}$ was assumed. The simulated optical response shows $t_{\text {on }}$ $=12.9 \mathrm{~ns}, t_{\mathrm{off}}=5.6 \mathrm{~ns}$, giving $f_{\max } \approx 54 \mathrm{MHz} \quad(\approx 18.5-\mathrm{ns}$ switching time) achievable in this particular bias case of

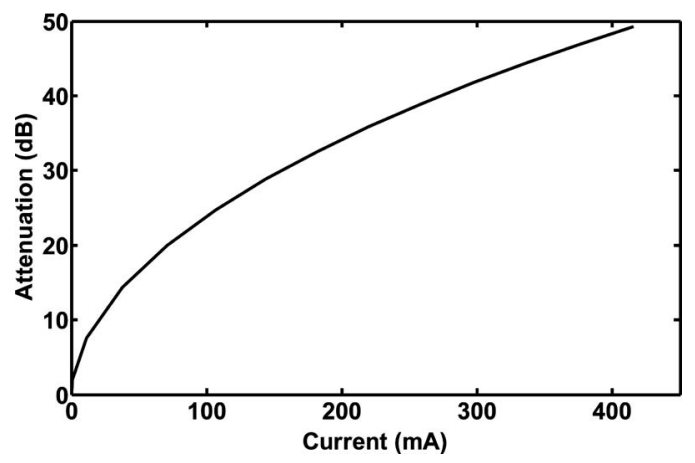

Fig. 8 Attenuation versus bias current of the ridge waveguide with $p n$ junction diode under forward bias for intensity (loss) modulation.

$V_{\text {off }}=-2.0 \mathrm{~V}$ and $V_{\text {on }}=2.0 \mathrm{~V}$. Up to $49.3-\mathrm{dB}$ modulation depth (i.e., extinction ratio) is attainable by this choice of biases.

The characteristic (static attenuation versus bias current) of the diode waveguide is shown in Fig. 8 By trading off some of the 18.5-ns switching time, one can achieve higher extinction ratios, which can be beneficial for noise suppression in the TDU.

To estimate the performance of the MZI-based Si switches (phase modulation), a 5-MHz (200-ns period) square-waveform electrical signal with $t_{\text {rise }}=t_{\text {fall }}=35.5 \mathrm{ps}$ is applied to the diodes. In a 0.5 -cm-long waveguide, a $\pi$ phase shift is achievable for $V_{\text {off }}=0.75 \mathrm{~V}$ and $V_{\text {on }}=0 \mathrm{~V}$, that is, $V_{\pi}=0.75 \mathrm{~V}$. An optical loss of $0.9 \mathrm{~dB}$ loss in the off state $(0.75 \mathrm{~V})$ is obtained. Figure 9 shows the transient response of phase shift under these conditions. The transient optical response shows $t_{\mathrm{on}}=6.2 \mathrm{~ns}, t_{\mathrm{off}}=24.2 \mathrm{~ns}$, indicating that $f_{\max } \approx 33 \mathrm{MHz}$ (or $\approx 30$-ns TDU switching time) is attainable. These initial design studies of the proposed silicon-photonics-based TDU indicate the road to achieving the desired numbers for loss, crosstalk, delay range, and switching speed.

\section{Conclusions}

Over the years, the stringent rf beamformer system requirements have impeded deployment of an optically controlled PAA. Silicon photonics is a promising technology for fulfilling this elusive goal. For the first time, to our knowledge, this paper proposes, analyzes, and simulates a silicon-
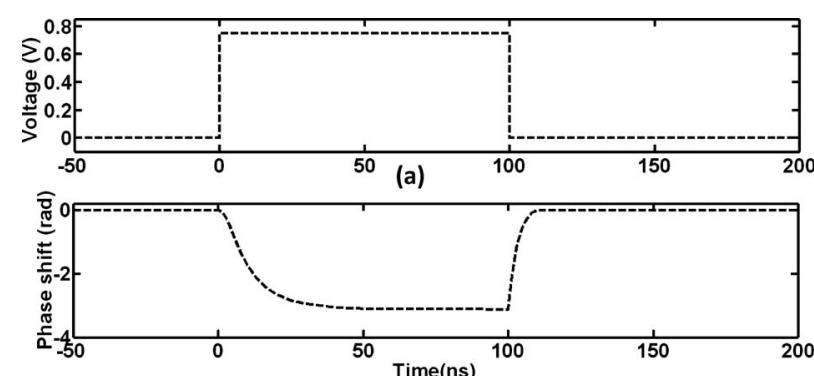

(b)

Fig. 9 Simulated switching results of a silicon phase modulator, showing: (a) applied electrical signal; (b) transient response for attaining $\pi$ phase shift. The result confirms that $\approx 30-n s$ switching time is attainable in the proposed switches. 
photonic TDU design to enable an element-level wideband beamformer ${ }^{42}$ The novel chip-scale TDU uses silicon 2 $\times 2$ switches, photonic delay lines, integrated Raman amplifiers, and VOAs. The reported design simulations suggest that it is feasible to obtain a high-performance allsilicon TDU that provides the low crosstalk $(50 \mathrm{~dB}$ optical), high speed ( $<1 \mu \mathrm{s}$ ), long delays (up to $2.5 \mathrm{~ns}$ ), and low insertion loss $(<6 \mathrm{~dB})$ required for an advanced beamforming TDU.

Future work relates to the fabrication of the proposed silicon TDU.

\section{References}

1. N. A. Riza, Ed., Selected Papers on Photonic Control Systems for Phased Array Antennas, SPIE Press, Bellingham, WA (1997).

2. N. Madamopoulos and N. A. Riza, "Demonstration of an all-digital 7-bit 33-channel photonic delay line for phased-array radars," Appl. Opt. 39, 4168-4181 (2000).

3. R. A. Soref, "Fiber grating prism for true time delay beam steering," Fiber Integr. Opt. 15, 325-333 (1996).

4. A. P. Goutzoulis, D. K. Davies, and J. M. Zomp, "Prototype binary fiber optic delay line," Opt. Eng. 28, 1193-1202 (1989).

5. D. Dolfi, P. Joffre, J. Antoine, J. P. Huignard, D. Philippet, and P. Granger, "Experimental demonstration of a phased-array antenna optically controlled with phase and time delays," Appl. Opt. 35, 52935300 (1996)

6. R. D. Esman, Y. Frankel, J. L. Dexter, L. Goldberg, M. G. Parent, D. Stilwell, and D. G. Cooper, "Fiber-optic prism true time delay antenna feed," IEEE Photonics Technol. Lett. 11, 1347-1349 (1993).

7. N. A. Riza and D. L. Polla, "Micromechanical fiber-optic switches for optical networks," in Integrated Optics and Microstructures OE/ Fibers Conf., Proc. SPIE 1793, 108-126 (1993).

8. G. A. Magel, T.-H. Lin, L. Y. Pang, and W. R. Wy, "Integrated optic switches for phased-array applications based on electrostatic actuation of metallic membranes," in Optoelectronic Signal Processing for Phased Array Antennas IV, B. M. Hendrickson, Ed., Proc. SPIE 2155, 107-113 (1994).

9. N. A. Riza and S. Sumriddetchkajorn, "Fault tolerant polarization insensitive photonic delay line architectures using two dimensional digital micromirror devices," Opt. Commun. 160, 311-320 (1999).

10. V. Kaman, X. Zheng, R. J. Helkey, C. Pusarla, and J. E. Bowers, "A 32-element 8-bit photonic true-time-delay system based on a 288 $\times 288$ 3-D MEMS optical switch," IEEE Photonics Technol. Lett. 15, 849-851 (2003).

11. J.-D. Shin, B.-S. Lee, and B.-G. Kim, "Optical true time-delay feeder for X-band phased array antennas composed of $2 \times 2$ optical MEMS switches and fiber delay lines," IEEE Photonics Technol. Lett. 16, 1364-1366 (2004).

12. N. A. Riza and S. Sumriddetchkajorn, "Micromechanics-based wavelength-sensitive photonic beam control architectures and applications," Appl. Opt. 39, 919-932 (2000).

13. N. A. Riza, M. A. Arain, and S. A. Khan, "Analog-digital variable fiber-optic delay line," J. Lightwave Technol. 22, 619-624 (2004).

14. E. J. Murphy, T. F. Adda, W. J. Minford, R. W. Irvin, E. I. Ackerman, and S. B. Adams, "Guided-wave optical time delay network," IEEE Photonics Technol. Lett. 8, 545-547 (1996).

15. K. Horikawa, I. Ogawa, T. Kitoh, and H. Ogawa, "Silica-based integrated planar lightwave true-time-delay network for microwave antenna applications," in OSA Optical Fiber Conf. Proc., pp. 100-101 (1996).

16. B. Howley, Y. Chen, X. Wang, Q. Zhou, Z. Shi, Y. Jiang, and R. T. Chen, "2-bit reconfigurable true time delay lines using $2 \times 2$ polymer waveguide switches," IEEE Photonics Technol. Lett. 17, 1944-1946 (2005).

17. C. T. Sullivan, S. D. Mukheriee, M. K. H. Brenner, A. Gopinath, E. Kalweit, T. Marta, W. Goldberg, and R. Walterson, "Switched timedelay elements based on AlGaAs/GaAs optical waveguide technology at $1.32 \mu \mathrm{m}$ for optically controlled phased array antennas," in Optical Technology for Microwave Applications IV and Optoelectronic Signal Processing for Phased-Array Antennas III, S.-K. Yao and B. M. Hendrickson, Eds. Proc. SPIE 1703, 264-271 (1992).

18. J. Stulemeijer, F. E. van Vliet, K. W. Benoist, D. H. P. Maat, and M. K. Smit, "Compact photonic integrated phase and amplitude controller for phased-array antennas," IEEE Photonics Technol. Lett. 11, 122-124 (1999).

19. N. A. Riza, "Switchless hybrid analog-digital variable optical delay line for rf signal processing," Opt. Eng. 48, 035005 (2009).

20. R. A. Minasian and K. E. Alameh, "High capacity optical beam form- ing for phased arrays with fiber gratings and frequency conversion for beat noise control," Appl. Opt. 38, 4665-4670 (1999).

21. A. Molony, L. Zhang, J. A. R. Williams, I. Bennion, C. Edge, and J. Fells, "Fiber Bragg-grating true time-delay systems: discrete-grating array 3-bit delay lines and chirped-grating 6-bit delay lines," IEEE Trans. Microwave Theory Tech. 45, 1527-1530 (1997).

22. J. L. Cruz, B. Ortega, M. V. Andres, B. Gimeno, D. Pastor, J. Capmany, and L. Dong, "Chirped fiber Bragg gratings for phased array antennas," Electron. Lett. 33, 545-546 (1997).

23. J. L. Corral, J. Marti, S. Regidor, J. M. Foster, R. Laming, and M. J. Cole, "Continuously variable true time-delay optical feeder for phased-array antenna employing chirped fiber gratings," IEEE Trans. Microwave Theory Tech. 45, 1531-1536 (1997).

24. B. Ortega, J. L. Cruz, J. Capmany, M. V. Andres, and D. Pastor, "Variable delay line for phased-array antenna based on a chirped fiber grating," IEEE Trans. Microwave Theory Tech. 48, 1352-1360 (2000).

25. Y. Liu, J. Yang, and J. Yao, "Continuous true-time-delay beamforming for phased array antenna using a tunable chirped fiber grating delay line," IEEE Photonics Technol. Lett. 14, 1172-1174 (2002).

26. N. A. Riza, "High-speed high-isolation $2 \times 2$ fiber-optic switch for wideband radar photonic beamforming controls," J. Lightwave Technol. 26, 2500-2505 (2008).

27. J. P. Lorenzo and R. A. Soref, "1.3 $\mu \mathrm{m}$ electro-optic silicon switch," Appl. Phys. Lett. 51, 6-8 (1987).

28. B. Jalali and S. Fathpour, "Silicon photonics," J. Lightwave Technol. 24, 1400-1415 (2006)

29. S. Yegnanarayanan, P. D. Trinh, F. Coppinger, and B. Jalali, "Compact silicon-based integrated optic time delays," IEEE Photonics Technol. Lett. 9, 634-635 (1997).

30. B. Little, S. Chu, W. Chen, W. Chen, J. Hryniewicz, D. Gill, O. King, F. Johnson, R. Davidson, K. Donovan, and J. Gibson, "Compact optical programmable delay lines with fast thermo-optic switching and output power balancing," in Avionics Fiber-Optics and Photonics, pp. 68-69, IEEE (2006)

31. Kotura Corporation, www.kotura.com

32. C. L. Schow, L. Schares, S. J. Koester, G. Dehlinger, R. John, and F. E. Doany, "A 15-Gb/s 2.4-V optical receiver using a Ge-on-SOI photodiode and a CMOS IC," IEEE Photonics Technol. Lett. 18, 1981-1983 (2006)

33. P. Dainesi, L. Thevanaz, and P. Robert, " $5 \mathrm{MHz} 2 \times 2$ optical switch in silicon on insulator technology using plasma dispersion effect," in 27th Eur. Conf. on Optical Communication, vol. 2, pp. 132-133 (2001)

34. P. D. Trinh, S. Yegnanarayanan, F. Coppinger, and B. Jalali, "Siliconon-insulator (SOI) phased-array wavelength multi/demultiplexer with extremely low polarization sensitivity," IEEE Photonics Technol. Lett. 9, 940-942 (1997).

35. A. Liu, H. Rong, R. Jones, O. Cohen, D. Hak, and M. Paniccia, "Optical amplification and lasing by stimulated Raman scattering in silicon waveguides," J. Lightwave Technol. 24, 1440-1455 (2006).

36. O. Boyraz and B. Jalali, "Demonstration of a silicon Raman laser," Opt. Express 12, 5269-5273 (2004).

37. H. Rong, R. Jones, A. Liu, O. Cohen, D. Hak, A. Fang, and M. Pannicia, "A continuous-wave Raman silicon laser," Nature (London) 433, 725-728 (2005)

38. S. Fathpour, K. K. Tsia, and B. Jalali, "Energy harvesting in silicon Raman amplifiers," Appl. Phys. Lett. 89, 061109 (2006).

39. B. Jalali, S. Fathpour, and K. K. Tsia, "Green silicon photonics," Opt. Photonics News 20, 18-23 (2009).

40. S. Fathpour and B. Jalali, "Energy harvesting in silicon optical modulators," Opt. Express 14, 10795-10799 (2006).

41. C. Gunn, "CMOS photonics for high-speed interconnects," IEEE MICRO 26, 58-66 (2006).

42. N. A. Riza and S. Fathpour, "Hybrid Design Variable RF Delay Line Using Silicon Photonics," US Patent Pending.

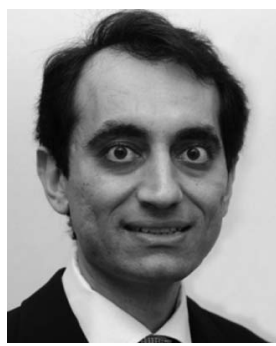

Sasan Fathpour is an assistant professor of optics at CREOL, The College of Optics and Photonics, at University of Central Florida, which he joined in 2008. He received his PhD degree in electrical engineering from the University of Michigan, Ann Arbor, in 2005 for his research on GaAs-based quantum dot lasers and spintronic light sources. He then joined the Electrical Engineering Department of UCLA as a postdoctoral fellow researching on nonlinear silicon photonics, and was a visiting assistant professor there in 2007. Before joining CREOL, he was a senior researcher at Ostendo Technologies, Inc., in Carlsbad, CA, working on nitride light 
sources for display applications. His current research interests include applications of silicon photonics to microwave photonics, photovoltaics, and telecommunications. Dr. Fathpour is the winner of the 2007 UCLA Chancellor's Award for Postdoctoral Research. He is a coauthor of more than 55 journal and conference publications and book chapters.

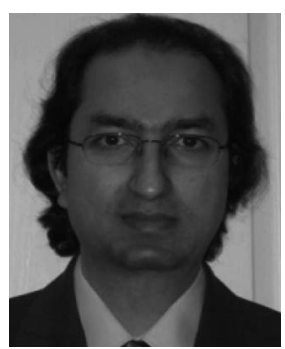

Nabeel A. Riza holds a doctorate from the California Institute of Technology. In January 2002, he became the first Caltech alumnus to be awarded the prestigious International Commission for Optics (ICO) Prize. He was co-awarded 2001 Ernst Abbe Medal from the Carl Zeiss Foundation, Germany. Riza's other awards include the 2007 IEEE Fellow Award, 1998 OSA Fellow Award, 1998 International Society for Optical Engineering (SPIE) Fellow Award, and 2008 Illinois Institute of Technology (IIT) Distinguished Alumni Professional
Achievement Award. In 2008 he was a Berthold Leibinger Innovation Prize nominee. After completing his PhD in 1989, Riza joined the General Electric (GE) Corporate Research and Development Center, New York, where he initiated and led the GE Optically Controlled Radar Project. In 1995, he joined CREOL, The College of Optics \& Photonics at the University of Central Florida, where he is a full professor and head of the Photonic Information Processing Systems Laboratory. For 2007-2008, he was selected as EU Erasmus Scholar Visiting Professor at the Delft University of Technology, The Netherlands. (Further information at http://pips.creol.ucf.edu.) 